
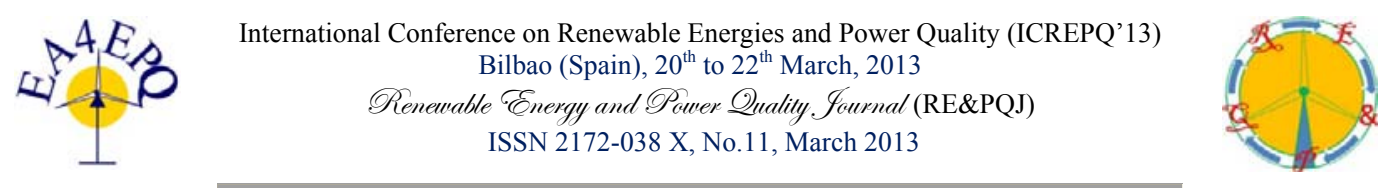

\title{
Verification tests of a novel ferroresonance detection technique
}

\author{
V. Valverde, G. Buigues, A.J. Mazón, I. Zamora, A. Etxegarai \\ Department of Electrical Engineering \\ Faculty of Engineering of Bilbao, UPV-EHU \\ Alameda de Urquijo s/n, 48013 Bilbao (Spain) \\ Phone/Fax number:+0034 946014172, e-mail: victor.valverde@ehu.es
}

\begin{abstract}
Ferroresonance is a complex electromagnetic phenomenon that affects voltage transformers. The appearance of the ferroresonant phenomenon provokes important oscillations that are responsible for serious thermal and dielectric overstresses, which may lead to catastrophic failures in the electrical power system. This paper analyzes the behaviour of a novel ferroresonance detection technique, based on artificial neural networks, through the results obtained in several verification tests conducted in a real voltage transformer.
\end{abstract}

\section{Key words}

Ferroresonance, Ferroresonance Detection, Voltage Transformer, Artificial Neural Networks

\section{Introduction}

Voltage transformers are devices particularly prone to ferroresonance phenomenon. This phenomenon is a special case of resonance that appears after transient disturbances (transient overvoltage, lightning overvoltage or temporary fault) or switching operations (transformer energizing or fault clearing), and its effects are characterized by high sustained overvoltages and overcurrents with maintained levels of current and voltage waveform distortion [1]. The practical measures that can be taken in order to mitigate the ferroresonance basically consist of introducing some losses into the system, in order to make the energy supplied by the power source insufficient to maintain this phenomenon. These losses can either be temporary (detection techniques) or permanent (prevention techniques). On the one hand, the latter may affect the efficiency of the installation in a considerable way, even provoking thermal failures under unbalanced situations. On the other hand, if the introduction of these losses is temporary, some sort of ferroresonance detection system would be necessary.

Nowadays, the current development level of this kind of detection systems is quite limited, although during the last years, the production of detection techniques and devices has started to be increased. In this context, Artificial Neural Network (ANN) theory emerges as an optimal solution to tackle the difficulties of the ferroresonance detection, as can be shown in the detection techniques presented in [2-3].
This paper analyzes the behavior of the ferroresonance detection technique presented in [2] through the results of several verification tests conducted in a real voltage transformer.

\section{Ferroresonance detection techniques}

Since ferroresonance problem in voltage transformers was first analysed by L.N. Robinson in 1915 [4], several authors have studied the possibilities of preventing the ferroresonant oscillations in this kind of transformer [5-6]. Nowadays, the most common measure against ferroresonance is the permanent inclusion of a damping circuit (ferroresonance suppression circuit) in the secondary winding of the transformer. The final design of this ferrorresonance suppression circuit depends on the transformer type and the electric configuration of the power system [7-8]. Figure 1 shows the typical elements included in the design of a ferroresonance suppression circuit [9].

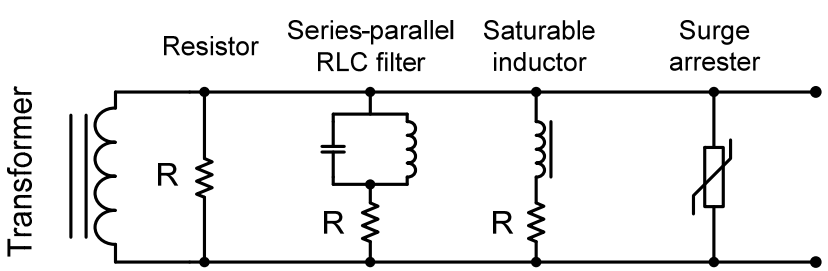

Figure 1. Basic circuits for ferroresonance suppression [9].

Furthermore, some techniques based on the selectively connection of the damping resistor have recently been developed. This way the damping circuit is only connected under ferroresonance situation and its impact on the efficiency of the installation is reduced. Within this field of ferroresonance detection, there are currently three main lines of research, depending on the criteria used to detect this phenomenon: saturation analysis [5,10-11], overvoltage analysis [12-13] and current/voltage waveform analysis [2-3,14].

On one hand, the first line of research is based on the use of an additional saturable inductor, whose saturation point has a slightly lower value than the voltage transformer's one. Consequently, the additional inductor will get 
saturated before the voltage transformer, leading to an early detection of the phenomenon. Its effectiveness depends on the degree of accuracy achieved in the design of the saturable inductor. On the other hand, the presence of overvoltages over a specified threshold may be implemented as an activation trigger for the connection of a damping circuit. This technique largely depends on the selected threshold value and does not take into account the type of event that causes the overvoltage.
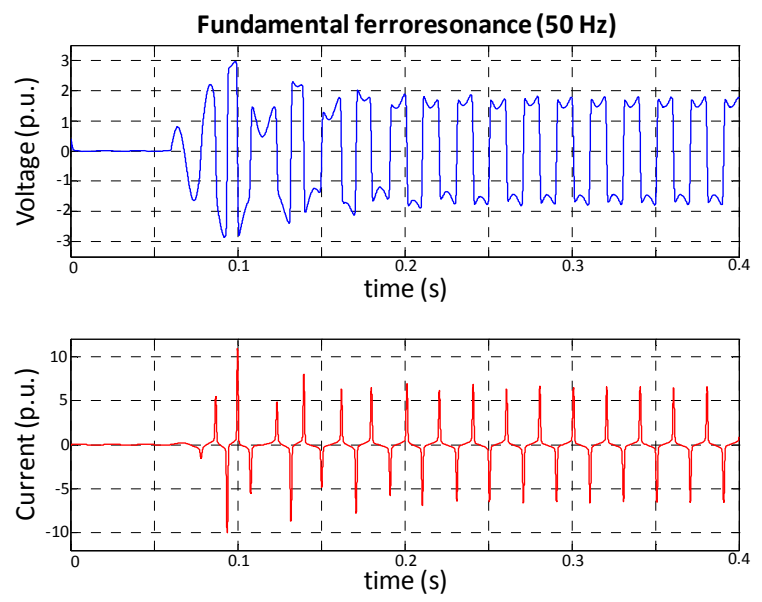

Figure 2. Voltage and current waveform of fundamental ferroresonance.

Finally, the last line of research is based on the characteristic waveforms that adopt the ferroresonant oscillations (Fig. 2). These characteristic oscillations of a ferroresonant situation may allow its identification by recognizing the disfigurement of voltage and current waveforms. In this way, for the last decade, a few methods of ferroresonance identification have been presented by combining the use of Wavelet Transform (WT) and ANN theories [3]. These methods based their performance on extracting a distinctive feature of the signal, in order to be classified by different ANNs.

\section{Novel ferroresonance detection technique}

The detection technique presented in [2] uses a MultiLayer Perceptron (MLP) network to identify the waveform of the voltage signal under ferroresonant conditions. This MLP network is trained to analyze the direct sampling of a full-cycle data window of the voltage signal at the fundamental frequency $(50 \mathrm{~Hz})$.

The detection system is configured to process the voltage signal provided by the secondary winding of the voltage transformer. Thus, it collects 32 samples/cycle, having a new sample every $0.625 \mathrm{~ms}$. These samples are stored in a 32-element vector, which is the input of the ANN after a conditioning stage (scale down the samples in a range between -1 and 1), until a data window is completed. The ANN processes these 32 data and provides a " $0 / 1$ " response (non-ferroresonant/ferroresonant window) depending on its interpretation (Fig. 3).

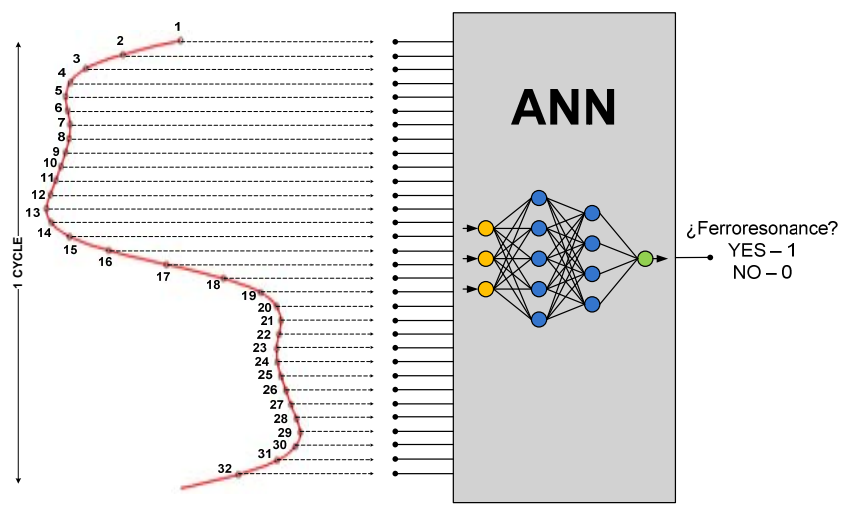

Figure 3. Network structure for ferroresonance detection

Once a response has been achieved, the process starts all over again by analyzing the next data window. This process is equivalent to moving the data window every $0.625 \mathrm{~ms}$, which is the time distance between two consecutive samples.

During the training process, the Levenberg-Marquardt learning algorithm is used. 1,640 different neural network architectures have been trained, varying the number of neurons of the first hidden layer from 1 to 40 and the neurons of the second hidden layer from 0 to 40 . Each network architecture has been tested several times, due to the randomness of each training.

After the training and verification process, the network architectures with better responses correspond to networks with 32 neurons in the input layer, a large number of neurons in the first hidden layer, a few neurons in the second hidden layer and one neuron in the output layer. Whereas the sigmoid activation function has been implemented in the neurons of the hidden layers, the identity function has been applied to the neuron of the output layer.

\section{Verification tests}

In order to verify the behaviour of the novel ferroresonance detection technique, several simulations and laboratory tests have been conducted using a real voltage transformer valid for outdoor service. Its general characteristics are $20 \mathrm{kV} / 230 \mathrm{~V}(50 \mathrm{~Hz}), 50 \mathrm{VA}$.

The artificial neural network selected during the tests has an architecture of 32 neurons in the input layer, 30 neurons in the first hidden layer, 2 neurons in the second hidden layer and one neuron in the output layer. Previously, this network has been tested by means of software simulation providing highly satisfactory results.

Once the software verification has been performed, several laboratory tests have been conducted in a specialized laboratory. The voltage transformer has been tested under no-load conditions using different switching times in order to force the ferroresonance to appear. Shortcircuit situations have also been provoked to force the phenomenon. A damping resistor has been used to damp 
the ferroresonant oscillations. Additionally, a series fuse has been installed in order to protect the circuit.

Figure 4 shows a basic schematic diagram of how the laboratory tests have been conducted. It includes a $6.7 \mathrm{nF}$ (50 kV of breakdown voltage limit) series capacitor and a $100 \Omega$ resistor (damping resistor).

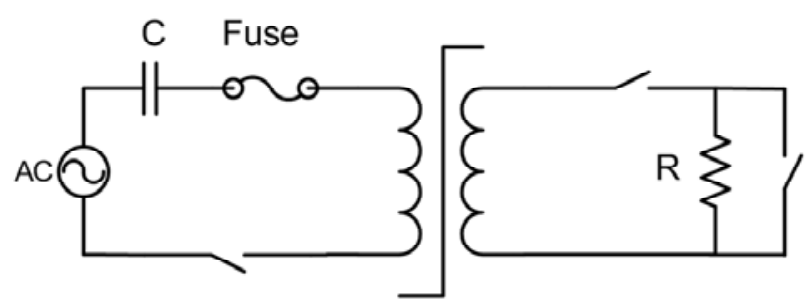

$20 \mathrm{kV} / 230 \mathrm{~V}$

Figure 4. Basic schematic diagram of the laboratory set-up.

In order not to jeopardise the integrity of the capacitor due to the ferroresonant overvoltages, the source voltage level has been limited to $28.5 / \sqrt{ } 3 \mathrm{kV}$ (Fig. 5)

Below, four of the conducted laboratory tests are analyzed.
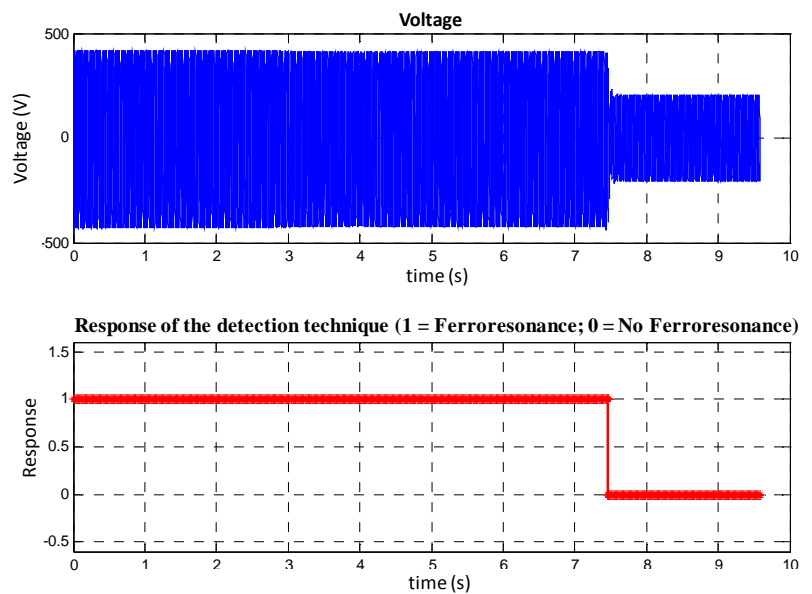

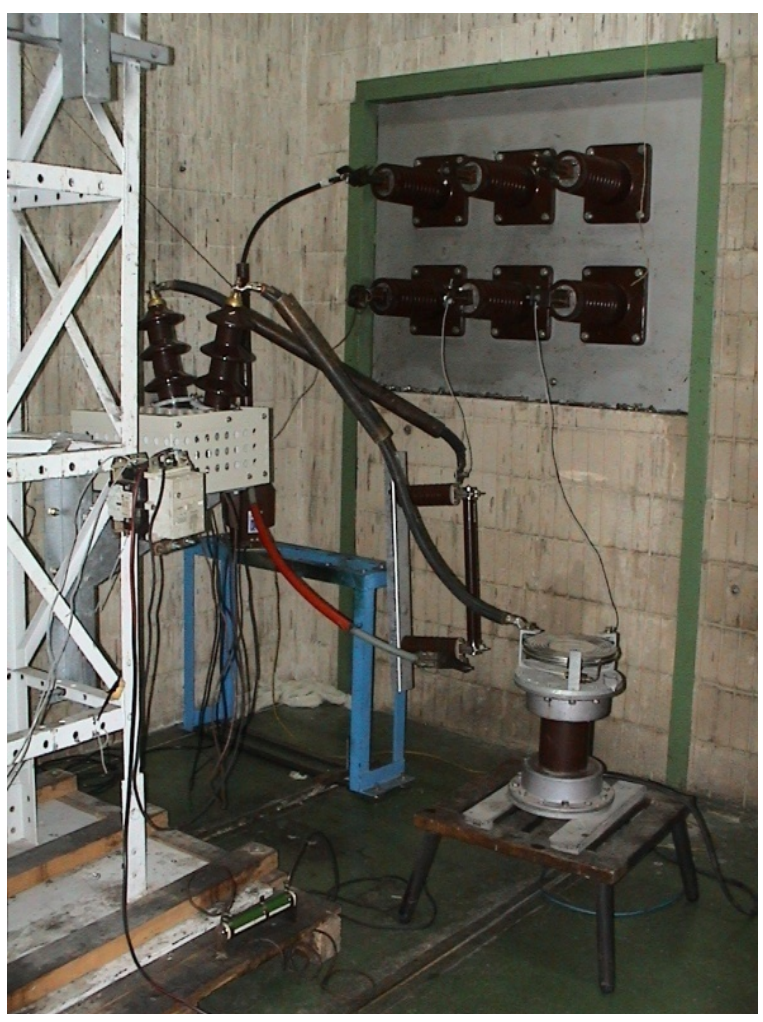

Figure 5. Laboratory set-up
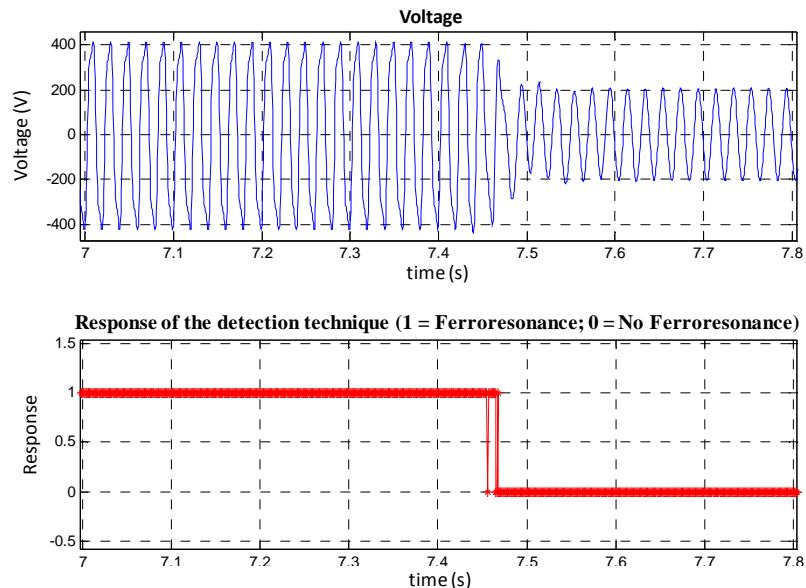

Figure 6. Response of the novel detection technique during verification test \#1
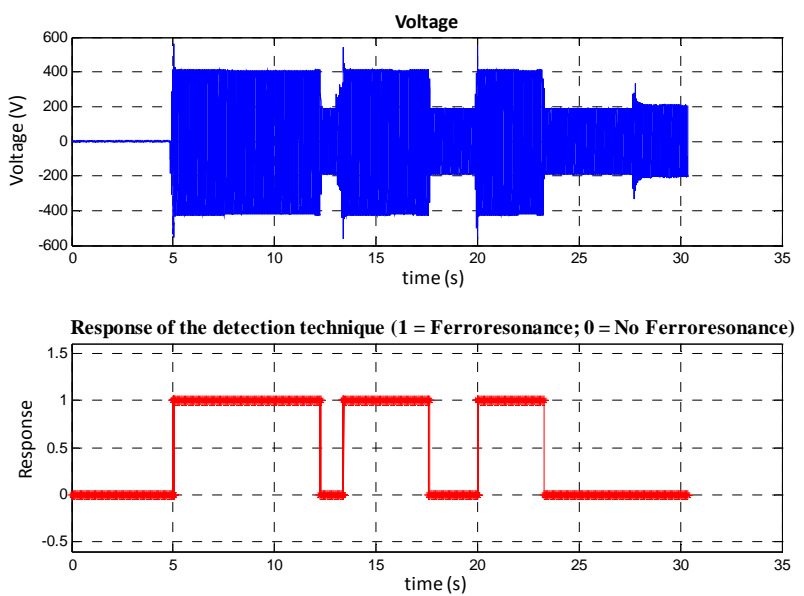
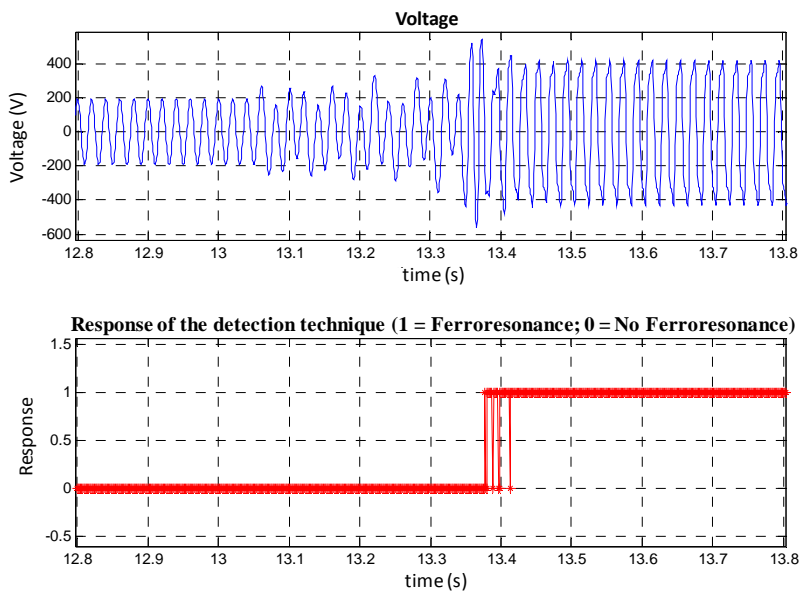

Figure 7. Response of the novel detection technique during verification test \#2 

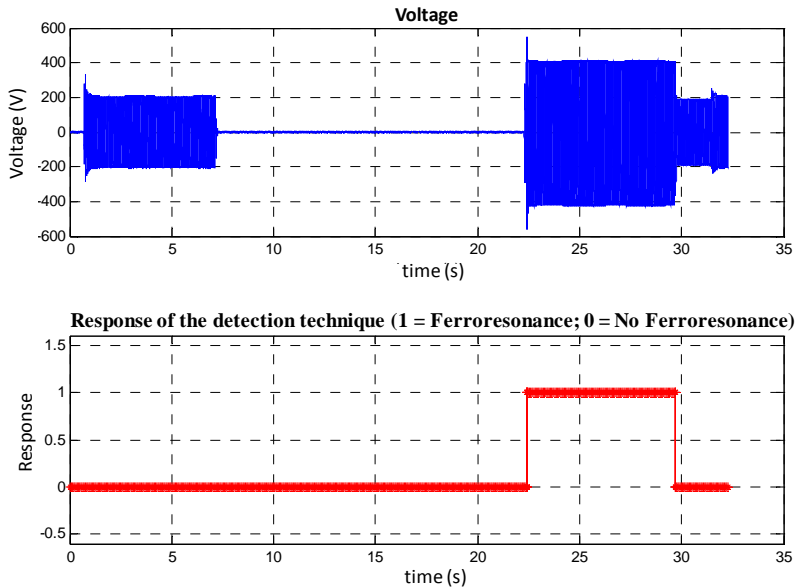
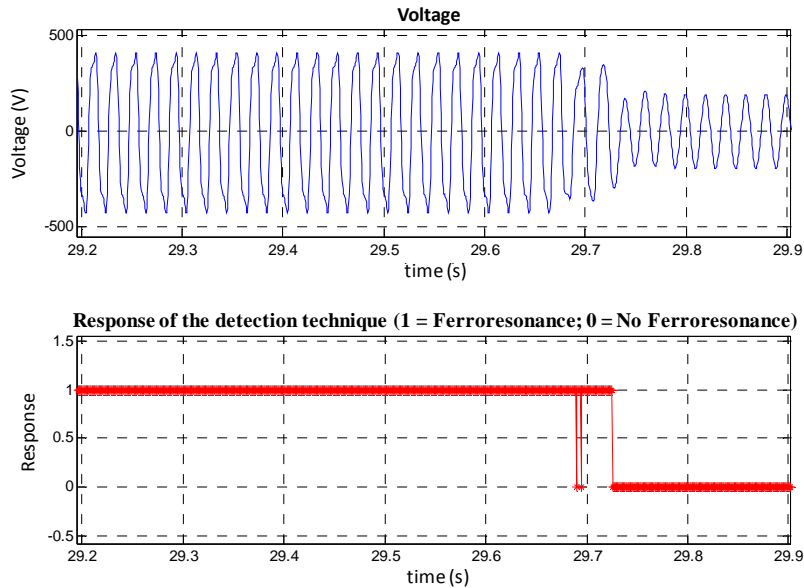

Figure 8. Response of the novel detection technique during verification test \#3
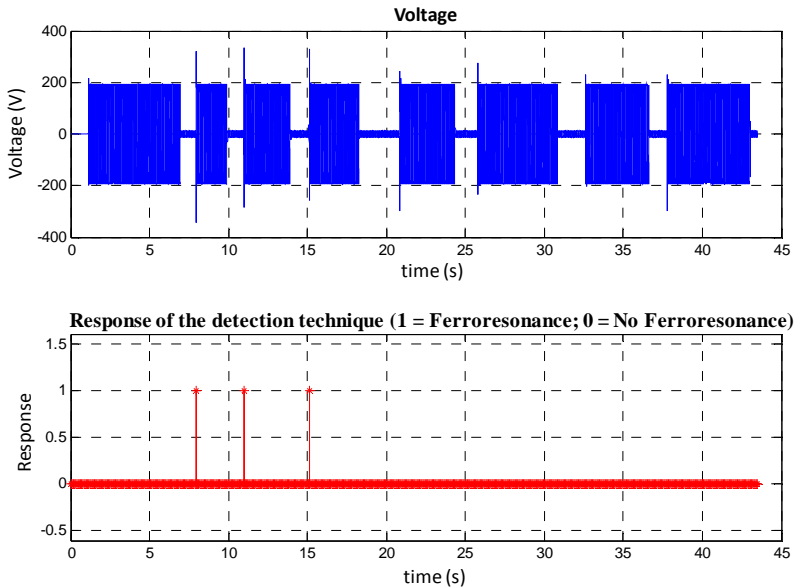
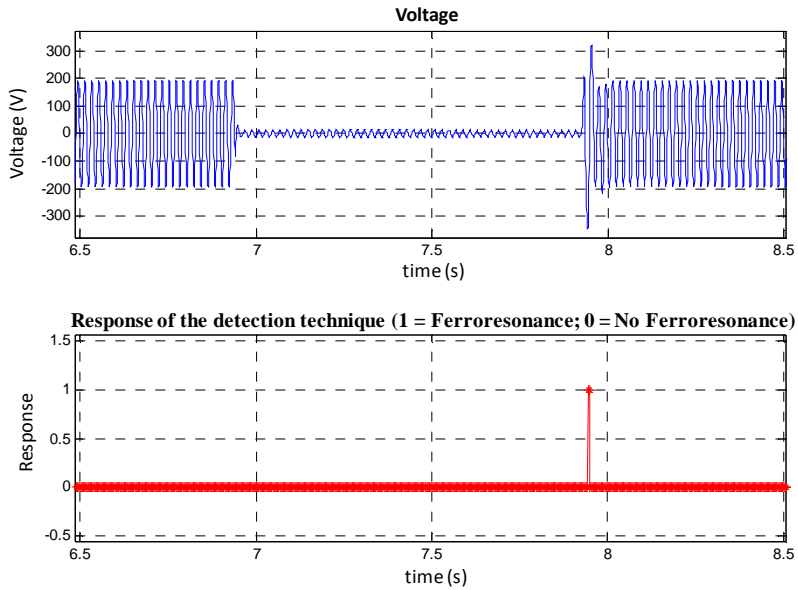

Figure 9. Response of the novel detection technique during verification test \#4

\section{A. Verification test \#1}

Firstly, the transformer is energized under no-load conditions, establishing a ferroresonant state at the fundamental frequency. Once the novel detection technique is applied, the ferroresonance is detected. Approximately at the second 7.5 , it is subsequently damped using a $100 \Omega$ resistor in the secondary winding (Fig. 6).

\section{B. Verification test \#2}

After 5 seconds, a first energization of the transfomer under no-load conditions provokes ferroresonant oscillations at the fundamental frequency. The novel detection technique works perfectly and, approximately at the second 12 , the $100 \Omega$ resistor is connected damping the phenomenon. From this moment on, the $100 \Omega$ resistor is consecutively connected and disconnected several times, leading to an intermittent appearance of the ferroresonance.
In every case, the novel detection technique successfully detects it (Fig. 7). Around the second 27.5, the $100 \Omega$ resistor is disconnected for the last time but the transformer does not reach a ferroresonant state. This is because the appearance of the phenomenon is strongly dependent on the exact moment of time in which the connection/disconnection is accomplished. Even in this last case, the novel detection technique works perfectly identifying a non-ferroresonant state.

\section{Verification test \#3}

Since a first energization of the transfomer under no-load conditions does not provoke the appearance of the ferroresonance, the transformer is disconnected for around 15 seconds. Then it is connected again and the ferroresonant oscillations appear. Just before the second 30 , the $100 \Omega$ resistor is connected damping the phenomenon. Almost 2 seconds later, this resistor is disconnected without the appearance of the ferroresonant phenomenon (Fig. 8). Through the whole process, the 
novel detection technique succeeds in identifying the ferroresonance every time it appears.

\section{Verification test \#4}

The transformer is energized under load conditions (100 $\Omega$ resistor is connected) and, during 45 seconds, its secondary winding is shortcircuited several times (Fig 9). This is performed in order to get a transient that triggers a ferroresonant state but none of the shortcircuits establishes the phenomenon. The novel detection technique successfully identifies the whole process as nonferroresonant.

\section{Conclusion}

This paper presents the results provided in several verification tests for a ferroresonance detection technique based on Artificial Neural Networks. During the conducted tests a real voltage transformer has been tested under ferroresonance conditions. It is shown that the novel detection technique clearly identifies the ferroresonance, as well as the non-ferroresonant oscillations providing a continuous output value of 1 or 0 respectively.

\section{Acknowledgement}

The work presented in this paper has been supported, partially, by the Basque Government (Ref. IT532-10) and by the University of the Basque Country UPV/EHU (UFI 11/28).

\section{References}

[1] Ferracci, P.,"Ferroresonance", Groupe Schneider: Cahier technique no 190, March 1998.

[2] V. Valverde, J. Mazón, G. Buigues, I. Zamora "Ferroresonance suppression in voltage transformers" Przegląd Elektrotechniczny (Electrical Review), Vol 88 (1a), pp. 137-140, Jan 2012

[3] Mokryani, G., Haghifam, M. R. and Esmailpoor, J.: "A novel technique for ferroresonance identification in distribution networks", International Journal of Electrical, Computer and System Engineering, 2007, vol. 1, pp. 103108.

[4] L. N. Robinson, "Phenomena Accompanying Transmission with Some Types of Star Transformer Connections", American Institute of Electrical Engineers, Transactions of the, vol. XXXIV, pp. 2183-2195, 1915.

[5] Craenenbroeck, T. V., Dommelen, D. V. and Janssens, N.: "Damping circuit design for ferroresonance in floating power systems", European Transactions on Electrical Power, May/June 2000, vol. 10, pp. 155-159.

[6] M. Sanaye-Pasand, A. Rezaei-Zare, H. Mohseni, S. Farhangi, and R. Iravani, "Comparison of performance of various ferroresonance suppressing methods in inductive and capacitive voltage transformers", in Power India Conference, 2006 IEEE, 2006, p. 8 pp.

[7] D. A. N. Jacobson, "Examples of ferroresonance in a high voltage power system", in Power Engineering Society General Meeting, 2003, IEEE, 2003, pp. 1206-1212.

[8] V. Valverde, G. Buigues, A. J. Mazón, I. Zamora, I. Albizu "Ferroresonant Configurations in Power Systems" International Conference on Renewable Energies and Power
Quality (ICREPQ) Santiago de Compostela (Spain), 28th to 30th March, 2012.

[9] J. Horak, "A review of ferroresonance", in Protective Relay Engineers, 2004 57th Annual Conference for, 2004, pp. 1-29.

[10] D. Shein and S. Zissu, "Domains of ferroresonance occurrence in voltage transformers with or without damping reactors", in Electrical and Electronics Engineers in Israel, 1995., Eighteenth Convention of, 1995, pp. 1.5.2/1-1.5.2/5.

[11] Piasecki, W., Stosur, M., Florkowski, M., Fulczyk, M. and Lewandowski, B.: "Mitigating ferroresonance in $H V$ inductive transformers", presented at the International Conference on Power Systems Transients, IPST'09, 2009, Kyoto, Japan.

[12] Sanaye-Pasand, M. and Aghazadeh, R.: "Capacitive voltage substations ferroresonance prevention using power electronic devices", presented at the International Conference on Power Systems Transients - IPST 2003, 2003, New Orleans, USA.

[13] W. Piasecki, M. Florkowski, M. Fulczyk, P. Mahonen, and W. Nowak, "Mitigating Ferroresonance in Voltage Transformers in Ungrounded MV Networks", Power Delivery, IEEE Transactions on, vol. 22, pp. 2362-2369, 2007.

[14] Mokryani, G., Siano, P. and Piccolo, A.: "Identification of ferroresonance based on S-transform and support vector machine", Simulation Modelling Practice and Theory, 2010, vol. 18, pp. 1412-1424. 